\title{
Calidad de vida en reconstrucción mamaria postmastectomía. Aplicación del instrumento Breast- ${ }^{\circledR}$ Quality of life in breast reconstruction after mastectotmy. Application of the Breast- $Q^{\oplus}$ instrument
}

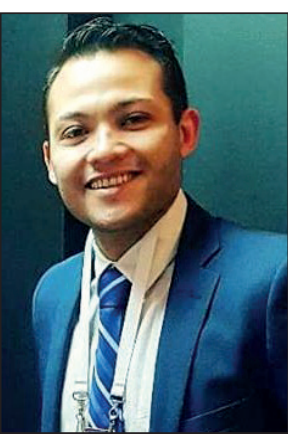

Gallegos Sierra C.
Resumen

Introducción y objetivo. A diferencia de los resultados oncológicos, no existen métodos estándar para evaluar los resultados estéticos. Muchos estudios han evaluado resultados cosméticos y calidad de vida después de la cirugía de cáncer de mama; los resultados fueron muy inconsistentes, tal vez debido al uso de evaluaciones subjetivas no confirmadas, al pequeño tamaño poblacional del estudio, al diseño retrospectivo y las diferencias en las medidas de calidad de vida.

El objetivo del presente estudio es determinar el estado de calidad de vida con el instrumento Breast- $\mathrm{Q}^{\circledR}$ (previamente autorizado por 2018 Mapi Research Trust $^{\dagger}$ y validado también para su aplicación en español) en mujeres sometidas a reconstrucción mamaria postmastectomía entre 2013-2018.

Material y método. Estudio de cohorte que incluye pacientes con diagnóstico de cáncer de mama sometidas a reconstrucción mamaria. Medimos su calidad de vida con el instrumento Breast- $\mathrm{Q}^{\circledR}$ en su versión en español. Mostramos las variables cuantitativas como promedios y desviación estándar. Los datos cualitativos como frecuencias y porcentajes. El análisis del antes y después de la reconstrucción mamaria con la prueba de Wilcoxon. Tomamos como significativo un valor $\mathrm{p}<0.05$. Para el análisis de los datos utilizamos el paquete estadístico SPSS versión 22.

Resultados. La satisfacción en cuanto a sus pezones, tórax, la información referida y en general, fue superior a 75 puntos sobre 100. De manera interesante, también el bienestar psicológico estuvo por encima del 75 de promedio. Al comparar el antes y después de la satisfacción con sus senos y del bienestar sexual encontramos un aumento significativamente estadístico.

Conclusiones. La evaluación mediante el instrumento Breast- $\mathrm{Q}^{\circledR}$ entre nuestro grupo de estudio demostró que la reconstrucción mamaria mejora la calidad de vida de las pacientes sometidas a este procedimiento.

Palabras clave Cáncer de mama, Calidad de vida, Reconstrucción mamaria, Breast-Q ${ }^{\circledR}$

\section{Nivel de evidencia científica Recibido [esta versión] Aceptado}

5 Significativo

5 febrero/2019

25 noviembre/2019

Conflicto de intereses: Los autores declaran no tener ningún interés financiero relacionado con el contenido de este artículo.

Financiación: No hubo fuentes externas de financiación para este trabajo.

Background and objective. Unlike oncological results there are no standard methods to evaluate aesthetic results. Many studies have evaluated cosmetic results and quality of life after breast cancer surgery with very inconsistent results perhaps due to the use of unconfirmed subjective evaluations, the small size of the population study, the retrospective design of the studies and differences in measures of quality of life.

Our objective is to determine the quality of life status with Breast- $Q^{\circledR}$ instrument (previously authorized by 2018 Mapi Research Trust $^{\circ}$ and also validated for its application in Spanish), in women with post-mastectomy breast reconstruction in 2013-2018.

Methods. Cohort study including patients diagnosed with breast cancer who underwent breast reconstruction. Quality of life was measured by the Breast-Q ${ }^{\circledR}$ instrument in its Spanish version. The quantitative variables are shown as averages and standard deviation. Qualitative data such as frequencies and percentages. The analysis of the before and after breast reconstruction with the Wilcoxon test. A value $p<0.05$ was taken as significant. The statistical package SPSS version 22 was used to analyze the data.

Results. Satisfaction regarding their nipples, their thorax, the referred information, and in general is above 75 points out of 100 . Interestingly, psychological well-being is also above 75 on average. When comparing the before and after satisfaction with their breasts and sexual well-being, a statistically significant increase was found.

Conclusions. The evaluation using the Breast- $\mathrm{Q}^{\circledR}$ instrument among our study group showed that breast reconstruction improves the quality of life of patients undergoing this procedure.

Key words Breast cancer, Quality of life, Breast reconstruction, Breast- $Q^{\infty}$

\section{Level of evidence Received [this version] \\ Accepted}

5 Meaningfulness

5 February/2019

\footnotetext{
Residente del Servicio de Cirugía Plástica y Reconstructiva, Hospital Regional Dr. Valentín Gómez Farías, Zapopan, Jalisco, México.

** Departamento de Ciencias Médicas, Centro Universitario de la Costa, Universidad de Guadalajara y Clínica Hospital Puerto Vallarta ISSSTE, Puerto Vallarta, Jalisco, México.

*** Médico Adscrito del Servicio de Cirugía Plástica y Reconstructiva, Hospital Regional Dr. Valentín Gómez Farías, Zapopan, Jalisco, México.

**** Médico Adscrito del Servicio de Oncocirugía, Hospital Regional Dr. Valentín Gómez Farías, Zapopan, Jalisco, México.

***** Subdirector de Prevención y Protección a la Salud, ISSSTE, Alcaldía Tlalpan, Ciudad de México, México.
} 


\section{Introducción}

Están publicados múltiples avances en la Oncología médico-quirúrgica pero, a pesar de ellos, el cáncer de mama sigue siendo la primera causa de muerte por neoplasia en la mujer con cerca de 500.000 muertes cada año, un mal que no solo afecta a una población específica o vulnerable, sino que tal como reportan Wang y col., ${ }^{(1)}$ el $70 \%$ de dichas muertes ocurre en países en desarro1lo. Las tasas de incidencia tienen una variación considerable en todo el mundo; las más altas se encuentran en Europa y Norteamérica con cifras estandarizadas de 99.4/100.000 mujeres. ${ }^{(2)}$ En el sur y centro de América, excepto Brasil y Argentina con incidencias también altas, se ha reportado una incidencia de moderada a baja de esta enfermedad. ${ }^{(3)}$ Tal y como se advierte en la norma oficial mexicana (último reporte epidemiológico), ${ }^{(4)}$ en México el cáncer de mama es ya la neoplasia maligna más frecuente y se ha convertido en un problema de salud pública que exige el concurso de todas las esferas de la sociedad y de la comunidad médica para su control.

Considerando los avances médico-quirúrgicos, es importante establecer que la Cirugía Plástica ha ayudado a la mejora en la calidad de vida. La reconstrucción mamaria ha evolucionado de tal manera que sus resultados han sido cada vez mejores, no solo en las cuestiones estéticas, sino también a favor de lograr la menor morbilidad posible en cada paciente. A pesar de que la evaluación de la calidad de vida sigue siendo un dato cualitativo que representa una de las variables clínicas más importantes para la evaluación de resultados, al pertenecer al ámbito subjetivo, pocas veces es medida. En la actualidad, se cuenta con instrumentos de medición validados llamados Patient Reported Outcomes Measurements (PROM), que permiten objetivar el estado de salud directamente del paciente. De todos los instrumentos publicados en los últimos años, el Breast- ${ }^{\circledR}$ ha expresado de manera significativa una mayor sensibilidad y especificidad para medir la calidad de vida de las pacientes sometidas a una mamoplastia, ya sea de aumento, reducción o reconstrucción. Si bien es cierto que Danilla y col. ${ }^{(5)}$ establecen las bases para un protocolo que utiliza dicho instrumento, solo se avocan a la valoración de pacientes sometidas a reducción mamaria.

En lo que respecta a la calidad de vida posterior a una intervención de Cirugía Plástica, señalamos también el trabajo publicado por Aracil y col. ${ }^{(6)}$ en 2012 en esta misma revista, en el que elaboran el primer instrumento que tiene como finalidad evaluar la calidad de vida en pacientes sometidos a un procedimiento de Cirugía Plástica; si bien conjunta de manera metódica los diferentes procedimientos quirúrgicos, no establece una pauta solo para procedimientos reconstructivos, sino que evalúa cirugías estéticas y reconstructivas en general utilizando el instrumento llamado $\mathrm{Ca}-\mathrm{CiP} 31$ que contiene evaluaciones psicométricas y también toma elementos del instrumento Breast- $Q^{\circledR}$.

En este sentido, cabe mencionar que después de haber realizado una exhaustiva búsqueda tanto en bases de datos nacionales como internacionales, principalmente bajo las palabras clave calidad vida y reconstrucción mamaria, no encontramos estudios que valoren bajo un instrumento estandarizado la calidad de vida en pacientes sometidas a reconstrucción mamaria, razón por la cual en el presente protocolo pretendemos evaluar los resultados relativos a dominios de calidad de vida con el instrumento Breast- $\mathrm{Q}^{\circledR}{ }^{(5,6)}$ asociados a reconstrucción mamaria secundaria al cáncer de mama en el Hospital Regional Dr. Valentín Gómez Farías, en Zapopan, Jalisco, México.

\section{Material y método}

Realizamos un estudio de cohorte en el que incluimos pacientes con diagnóstico de cáncer de mama sometidas a reconstrucción mamaria. Durante esta primera fase construimos y documentamos una base de datos de pacientes referidas al Servicio de Cirugía Plástica del Hospital Regional Valentín Gómez Farías en Zapopan (Jalisco, México) sometidas a mastectomía secundaria a cáncer de mama, e incluimos en el estudio final a todas aquellas que recibieron mamoplastia de reconstrucción de cualquier tipo (reconstrucción autóloga independientemente del tipo de colgajo, con o sin material protésico tipo implante y/o expansor, y con reconstrucción inmediata o diferida) en un periodo correspondiente a 5 años, entre 2013 y 2018.

Los datos se verificaron en el expediente clínico previa autorización del Servicio de Enseñanza e Investigación y Bioética del hospital. Cabe señalar que se protegieron escrupulosamente los datos personales (los aspectos éticos de la presente investigación se han establecido en los lineamientos y principios generales que el Reglamento de la Ley General de Salud en materia de investigación para la salud de nuestro país refiere). Solicitamos consentimiento informado por escrito tomando en cuenta: confidencialidad, sometimiento a Comité de Ética y Consideraciones Internacionales de Helsinki.

Una vez completada la base de datos se procedió a contactar a las pacientes para contestar el instrumento. Dicho instrumento fue Breast-Q ${ }^{\circledR}$ según Klassen y col. ${ }^{(7)}$ (previamente autorizado por 2018 Mapi Research Trust $^{\dagger}$ y validado también para su aplicación en español). Excluimos a aquellas pacientes que no quisieron atender a la llamada para la evaluación y no incluimos pacientes embarazadas 
o en lactancia, pacientes con otro diagnostico diferente a cáncer de mama, expediente clínico incompleto o perdido, datos incompletos (primarios), historial de radioterapia y pacientes menores de 18 años de edad. En cuanto al tiempo postoperatorio para aplicar el instrumento (tiempo trascurrido tras la reconstrucción mamaria) fue variado, pero establecimos un periodo medio de 6 meses, con un intervalo que transcurrió entre los 3 y los 12 meses.

El instrumento en concreto quedó con 39 ítems, los cuales se responden con una escala de Likert-Like pudiendo ser de 4 puntos: 1 "muy insatisfecho" a 4 "muy satisfecho"; 3 puntos: 1 " muy en desacuerdo" a 3 "de acuerdo"; 5 puntos: de 1 "en ningún momento" a 5 "todo el tiempo" (+/- no aplicable). Aplicamos el instrumento en físico tras explicación y firma del consentimiento informado.

Mostramos las variables cuantitativas como promedios y desviación estándar. Los datos cualitativos como frecuencias y porcentajes. El análisis del antes y después de la reconstrucción mamaria con la prueba de Wilcoxon. Tomamos un valor $\mathrm{p}<0.05$ como significativo. Para el análisis de los datos utilizamos el paquete estadístico SPSS versión 22.

\section{Resultados}

En el periodo señalado de 5 años evaluamos 268 pacientes con patología mamaria valoradas por el Servicio de Cirugía Plástica y Reconstructiva de nuestro hospital, 83 de las cuales sufrieron secuelas de cáncer de mama de las que 52 cumplieron con criterios para someterse a reconstrucción mamaria, pero solo 31 de estas atendieron la llamada para contestar nuestra encuesta y son las que presentamos. En la Tabla I presentamos las características demográficas de la muestra.

Tabla I. Características de la muestra.

\begin{tabular}{|lccc|}
\hline \multicolumn{1}{|c|}{ Variable } & \multicolumn{3}{c|}{ Valor } \\
\cline { 2 - 4 } & Media & Mínimo & Máximo \\
\hline Edad, años & $49 \pm 8$ & 41 & 57 \\
\hline Peso, kg & $67.2 \pm 7.3$ & 59.9 & 74.5 \\
Estatura, $\mathbf{m}$ & $1.6 \pm 0.05$. & 1.55 & 1.65 \\
IMC & $25.3 \pm 5.6$ & 19.7 & 30.9 \\
Escolaridad, $\mathbf{n}(\%)$ & & & \\
$\quad$ Preparatoria & & $10(32)$ & \\
$\quad$ Universidad & & $18(58)$ & \\
Otros & & $3(10)$ & \\
Estado civil, $\mathbf{n}$ (\%) & & \\
$\quad$ Casada \\
$\quad$ Divorciada \\
$\quad$ Otros
\end{tabular}

En cuanto al diagnóstico, encontramos mayor incidencia de cáncer de mama derecha con 16 casos re-

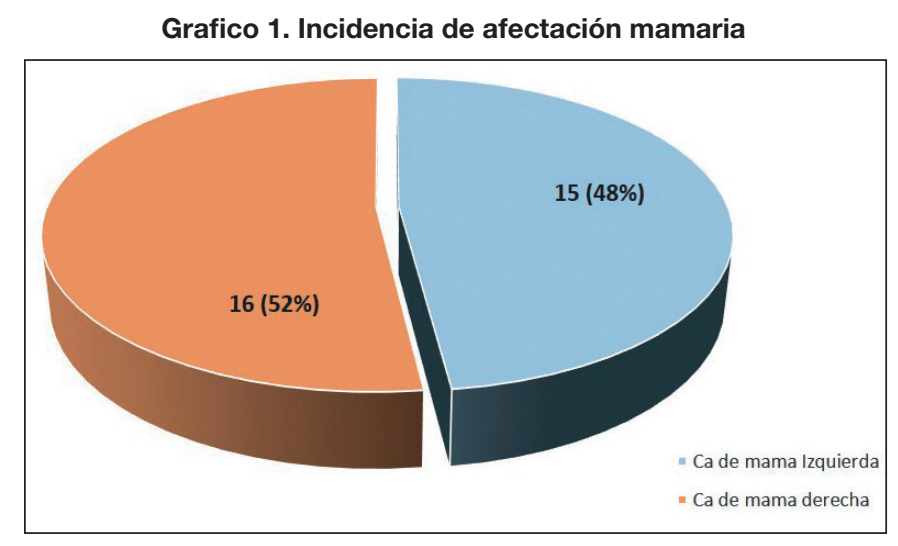

Grafico 2. Cirugía primaria realizada

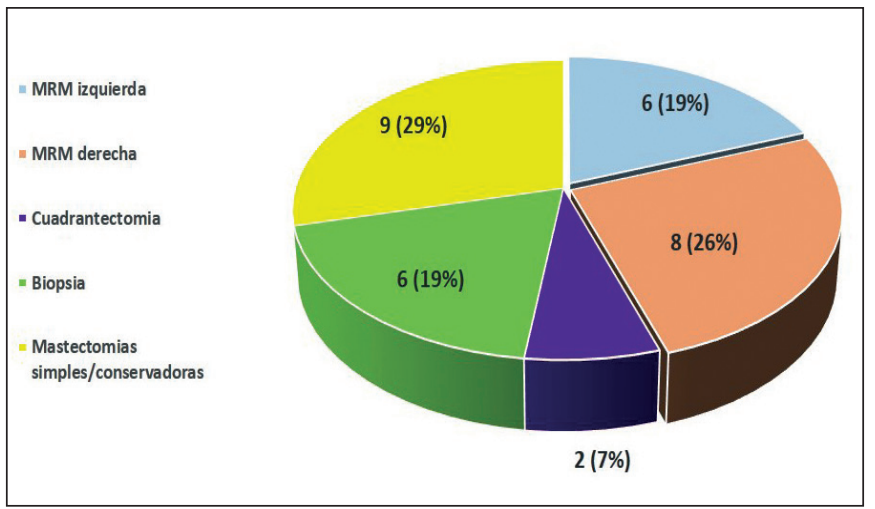

Grafico 3. Etapa clínica mayormente reportada

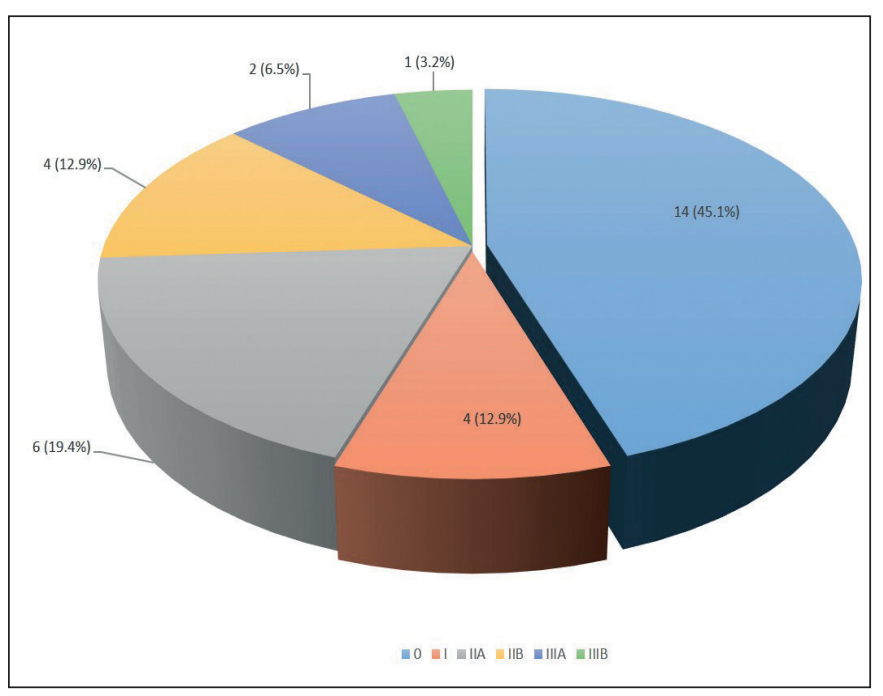

Grafico 4. Técnica de reconstrucción mamaria más utilizada

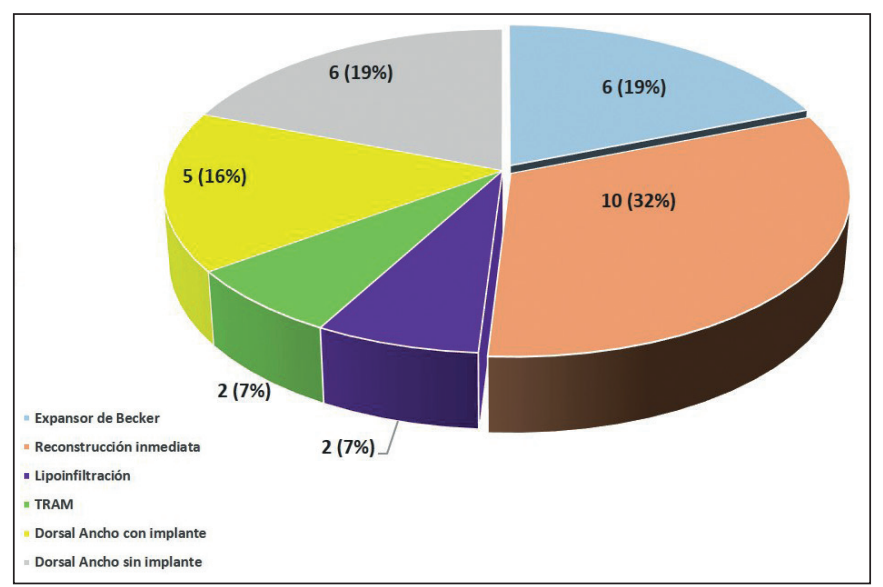


Grafico 5. Satisfacción postquirúrgica

\begin{tabular}{|l|l|l|l|l|l|}
\hline & Promedio & & & & P4.5 \\
\hline
\end{tabular}

Grafico 6. Comparación de satisfacción pre y postquirúrgica
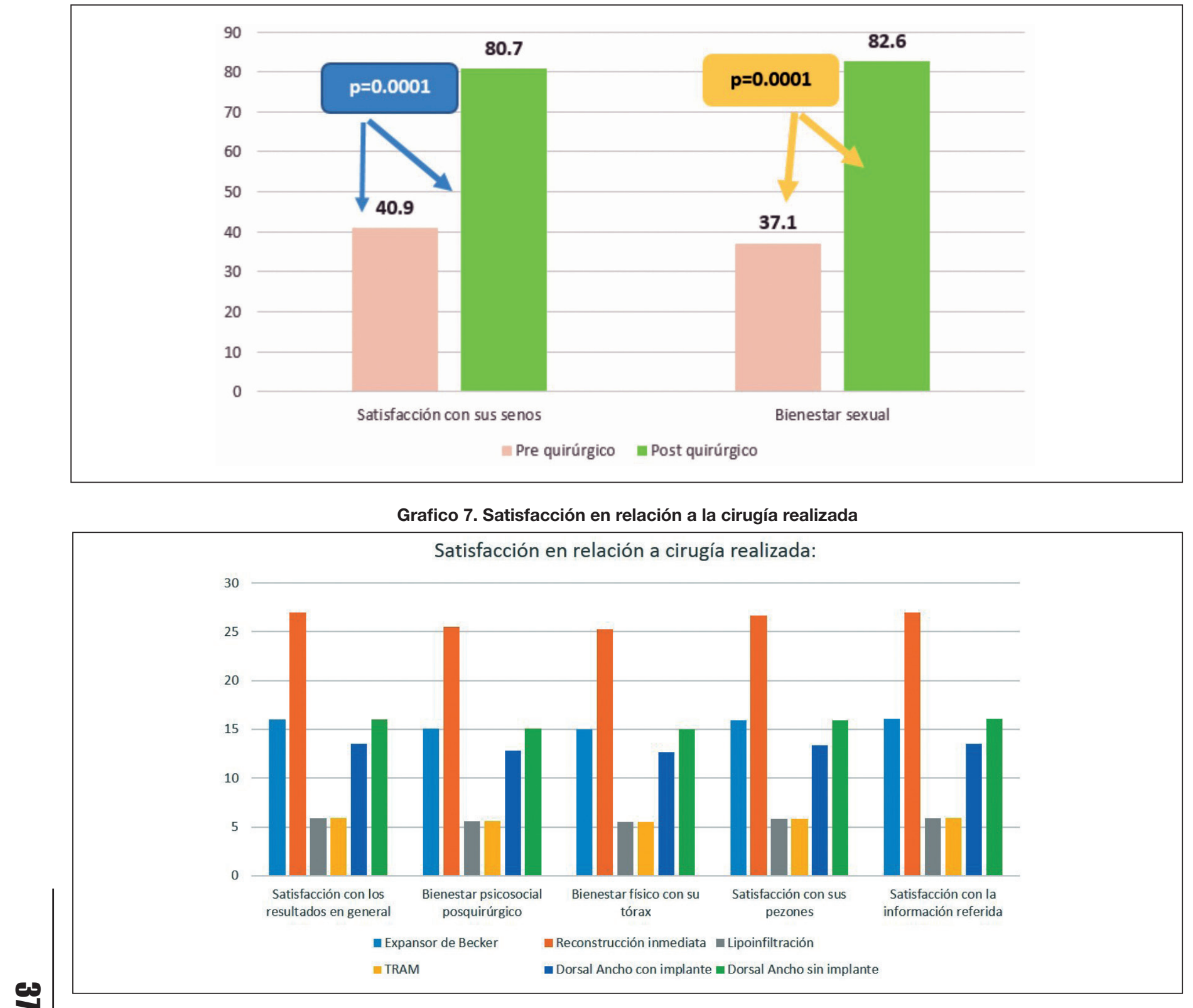
gistrados (52\%, Grafico 1). En promedio tuvieron 2 intervenciones (mínimo de 1 y máximo de 5), mientras que el procedimiento más realizado fue la mastectomía simple/conservadora en 9 pacientes (29\%), seguida por la mastectomía radical modificada (MRM) derecha en 8 pacientes (26\%) (Grafico 2).

La etapa clínica más reportada fue la 0 , registrando 14 pacientes (45.1\%), seguida por la IIA con 6 pacientes (19.4\%) (Gráfico 3). La técnica de reconstrucción más utilizada fue la inmediata con 10 pacientes $(32 \%)$, seguida por expansor de Becker en 6 pacientes (19\%) y dorsal ancho sin implante en otras 6 pacientes (19\%) (Grafico 4).

En relación al instrumento de calidad de vida, el Gráfico 5 presenta los promedios de las mediciones tras una calificación prequirúrgica (posterior a la mastectomía). Observamos que la satisfacción en cuanto a sus pezones, su tórax, la información referida y en general, está por encima de los 75 puntos sobre 100. De manera interesante, también el bienestar psicológico estuvo por encima del 75 en promedio. Al comparar los resultados tras la mastectomía y después de la reconstrucción, la satisfacción con sus senos y el bienestar sexual tuvo un aumento estadísticamente significativo en la muestra (Gráfico 6). Establecimos también que la reconstrucción inmediata tuvo una mayor satisfacción en general en comparación con las otras técnicas reconstructivas realizadas (Gráfico 7).

\section{Discusión}

El objetivo del presente trabajo fue determinar con el instrumento Breast- $Q^{\circledR}$ el estado de calidad de vida en mujeres con mastectomía por cáncer de mama más reconstrucción mamaria en el Servicio de Cirugía Plástica del hospital regional Dr. Valentín Gómez Farías en el periodo comprendido entre 2013-2018. Dicho hospital es una institución de tercer nivel conformada por equipos multidisciplinarios entre ellos Clínicas de Mama, Servicios de Oncología Médica y Quirúrgica, además del Servicio de Cirugía Plástica y Reconstructiva.

Hasta donde sabemos y en base a la bibliografía revisada de artículos sobre los resultados cosméticos y su afectación en la calidad de vida (CdV) de las pacientes mexicanas mastectomizadas por cáncer de mama, existen muy pocas estadísticas que establezcan la $\mathrm{CdV}$ en pacientes tras someterse a reconstrucción mamaria; si bien es cierto que se han evaluado algunas otras poblaciones con patologías de gigantomastia y sus resultados de $\mathrm{CdV}$ postreducción, tal como establecen Danilla y col. en $2016^{(5)}$ en un conjunto de pacientes chilenas, es importante establecer que la bibliografía revisada es semejante a nuestra población mexicana y que creemos que la $\mathrm{CdV}$ establece la culminación y el objetivo principales del tratamiento del cáncer de mama. Sin embargo, los estudios hasta la fecha han arrojado resultados inconsistentes. La evaluación con métodos estandarizados y validados pueden justificar los esfuerzos para mejorar los resultados cosméticos de esta intervención.

Considerando los avances médico-quirúrgicos, es importante establecer que la Cirugía Plástica ha contribuido a la mejora en la $\mathrm{CdV}$ de las pacientes mastectomizadas. La reconstrucción mamaria ha evolucionado de tal manera que sus resultados han sido cada vez mejores, no solo en las cuestiones estéticas, sino también a favor de provocar la menor morbilidad posible en cada paciente. Aunque cada persona experimenta cambios secundarios de una manera diferente, dichos cambios en la apariencia física a menudo conducen a modificaciones en la imagen corporal que conllevan estrés y ansiedad; incluso los mejores tratamientos tienen efectos secundarios, algunos de los cuales incluyen: fatiga, pérdida de cabello, movimientos de mano limitados a corto plazo, etc. Por todo ello, en nuestro estudio tratamos de evaluar resultados en términos de satisfacción con sus senos, bienestar psicosocial y bienestar sexual.

Si bien la reconstrucción mamaria tras mastectomía mejora la satisfacción de las pacientes según lo advierten Dean y col, también se sabe que la reconstrucción inmediata reduce la incidencia de depresión postoperatoria en comparación con las mujeres que se sometieron a una reconstrucción mamaria diferida ${ }^{(9)}$ Zhong y col. también demostraron una mejoría entre las mediciones preoperatorias y postoperatorias de satisfacción con el bienestar mamario, psicosocial y sexual en pacientes que se habían sometido a reconstrucción mamaria con colgajo de perforante de la arteria epigástrica inferior profunda (DIEP) y con colgajo miocutáneo de recto abdominal transverso (TRAM). ${ }^{(9)}$

La reconstrucción mamaria es un área de interés en curso, ya que las tasas de incidencia de cáncer de mama están aumentando en todo el mundo. ${ }^{(10,11)}$ En México, según el Consenso Mexicano sobre Diagnóstico y Tratamiento del Cáncer de Mama, ${ }^{(12)}$ en las últimas estimaciones del IARC (International Agency for Research on Cancer) indican que en el año 2013, en nuestro país, hubo 23.687 nuevos casos y fallecieron 5.902 pacientes.

Al-Ghazal y col. informan sobre una serie retrospectiva de pacientes en las que identifican mayor satisfacción y bienestar psicosocial en pacientes después de cirugía conservadora del complejo areola-pezón frente a mastectomía y reconstrucción. ${ }^{(12)}$ Sin embargo, su estudio utilizó medidas de resultado genéricas en lugar de específicas para el seno, como la escala de depresión de ansiedad del hospital, la escala de imagen corporal y la escala de autoestima de Rosenberg. 
Al igual que Elthair y Elder, ${ }^{(14,15)}$ encontramos que la estirpe etiológica más encontrada fue el adenocarcinoma ductal invasor (48\%) seguido del carcinoma ductal in situ (42\%), datos que son equiparable con los resultados mostrados en nuestro estudio en el que la estirpe etiológica más encontrada fue el adenocarcinoma ductal invasor (48\%) seguido del carcinoma ductal in situ (42\%). Además, en lo que respecta a la satisfacción con la cirugía realizada determinamos que la reconstrucción inmediata sobresale en comparación con las otras técnicas empleadas, tal y como se demuestra en el Gráfico 7 y coincidiendo con lo establecido por los autores anteriormente comentados. También realizamos comparación de nuestro estudio con otro artículo publicado por Ethair y col, ${ }^{(16)}$ en el cual se describe un estudio muy similar comparando los resultados de CdV entre mastectomía con reconstrucción, no solo utilizando el instrumento Breast-Q ${ }^{\circledR}$ sino también utilizando un instrumento llamado RAND-36 que consiste en 36 preguntas dirigidas a evaluar funcionamiento físico, emocional, vitalidad, salud mental, función social, dolor corporal y salud general de cada paciente, obteniendo resultados muy similares a los nuestros dado que al utilizar los 2 instrumentos anteriormente señalados, demuestran que la reconstrucción mamaria provoca una mejora en la $\mathrm{CdV}$ con un resultado estadísticamente significativo mayor (70.5; $\mathrm{p}=0.003$ ), con una puntuación para el bienestar psicosocial de una media del $66.6 \%$. Comparando con nuestro estudio, en el cual solo utilizamos el instrumento Breast- $Q^{\circledR}$, de manera interesante vimos que también el bienestar psicológico se encontró por encima del 75 en promedio. Al comparar el antes y después de la satisfacción con sus senos y del bienestar sexual, encontramos un aumento estadísticamente significativo en la muestra.

La aportación clínica de nuestro trabajo al prestador de servicios de salud va encaminada a establecer que la $\mathrm{CdV}$ deberá incluir todos aquellos aspectos que influyen en la salud física de la mujer, su estado psicológico, nivel de independencia, relaciones sociales y también la relación que tiene la persona con los elementos esenciales de su entorno. ${ }^{(17,18)}$ Creemos que la $\mathrm{CdV}$ de las pacientes puede mejorarse al determinar un vínculo entre los resultados estéticos objetivos y su satisfacción. Además, concentramos el presente estudio en los factores que afectan a la percepción del paciente de la imagen corporal y los métodos para mejorar su autopercepción. Como se puede apreciar en el Anexo 1, establecemos 4 tablas encaminadas a la evaluación del bienestar psicosocial, bienestar físico en relación a su tórax y principalmente a la satisfacción posterior a la reconstrucción mamaria. Las últimas 13 preguntas están encaminadas a la valoración sexual (6 pre- guntas) y a la percepción postreconstrucción (ultimas 7 preguntas); estos dos extractos del instrumento nos ayudan a esclarecer si de verdad las pacientes están satisfechas o no tanto con el ámbito sexual como con la reconstrucción en si. También si están satisfechas con la información recibida, con los resultados asociados a procedimientos reconstructivos exitosos y fallidos, junto con la probabilidad de fallo reconstructivo local y con si recibieron todo lo pertinente para realizar cualquier procedimiento reconstructivo secundario (de ser necesario) y el riesgo de que debieran someterse a una mastectomía simple, con sus resultados asociados, si surgieran complicaciones.

\section{Conclusiones}

En nuestro estudio de cohorte para evaluar la calidad de vida de pacientes postreconstrucción mamaria llevado a cabo en el hospital Dr. Valentín Gómez Farías, al comparar la satisfacción de antes y después con sus senos y su bienestar sexual encontramos un aumento en la escala de calidad de vida estadísticamente significativo en la muestra. Si bien otros estudios apuntan hacia la misma idea, no detectamos un estudio concluyente, posiblemente debido a que la evaluación de la calidad de vida sigue siendo un dato cualitativo difícil de medir y que a pesar de que representa una de las variables clínicas más importantes para la evaluación de resultados, al pertenecer al ámbito subjetivo pocas veces se mide.

Con el presente estudio proponemos el instrumento Breast- $Q^{\circledR}$ ya que hemos demostramos que la reconstrucción mamaria, en sus diversos procedimientos pero principalmente en la reconstrucción mamaria inmediata (cuando está indicada), se ha convertido en una opción viable para las pacientes mástectomizadas que desean mejorar su calidad de vida, principalmente en la apreciación de sus senos y de manera secundaria en su ámbito sexual. Definitivamente creemos que se necesitan estudios más extensos, con un mayor número de pacientes, adaptados a nuestra población; sin embargo también estamos seguros de que nuestro estudio será de utilidad y servirá tanto para nuevas investigaciones como para pacientes que estén en un periodo de indecisión.

\section{Dirección del autor}

\section{Dr. Cuauhtly Gallegos Sierra}

Departamento de Cirugía Plástica y Reconstructiva

Hospital Regional Dr. Valentín Gómez Farías, ISSSTE

Octavo piso

Av. Soledad Orozco 203, Col. El Capullo

C.P. 45150 Zapopan, Jalisco, México

Correo electrónico: cuauhtly1@gmail.com 
Bibliografía

1. Wang HT, Barone CM, Steigelman MB, et al. Aesthetic outcomes in breast conservation therapy. Aesthet Surg $J$ 2008; 28:165-170.

2. Schag CA, Ganz PA, Polinsky ML, et al. Characteristics of women at risk for psychosocial distress in the year after breast cancer. J Clin Oncol e Off J Am Soc Clin Oncol 1993; 11:783793.

3. Al-Ghazal SK, Fallowfield L, Blamey RW. Does cosmetic outcome from treatment of primary breast cancer influence psychosocial morbidity? Eur J Surg Oncol 1999; 25:571-573.

4. Norma Oficial Mexicana NOM-041-SSA2-2011 para la prevención, diagnóstico, tratamiento, control y vigilancia epidemiológica del cáncer de mama. Última modificación: 9 de junio de 2011. Disponible en: http://www.cenetec.salud.gob.mx/descargas/equipoMedico/normas/NOM_041_SSA2_2011.pdf

5. Danilla $\mathrm{S}$, Troncoso E, Ríos M.A, et al. ¿Qué factores influyen en la satisfacción de los pacientes sometidos a reducción mamaria? Análisis de un estudio de cohorte utilizando el instrumento Breast- ${ }^{\circledR}$. Revista Chilena de Cirugía 2017;69(1):28-34.

6. Aracil $\mathrm{K}$, et al. $\mathrm{Ca}-\mathrm{CiP} 31$ : Elaboración y validación del primer instrumento de calidad de vida en Cirugía Plástica en español. Cir. plást. iberolatinoam. 2012; 38(3):215-227.

7. Klassen AF, Pusic AL, Scott A, Klok J, Cano SJ. Satisfaction and quality of life in women who undergo breast surgery: a qualitative study. BMC Womens Health. 2009; 1(9):11

8. Dean C, Chetty U, Forrest AP. Effects of immediate breast reconstruction on psychosocial morbidity after mastectomy. Lancet 1983; 1:459-462.

9. Zhong T, McCarthy C, Min S, et al. Patient satisfaction and health-related quality of life after autologous tissue breast reconstruction: a prospective analysis of early postoperative outcomes. Cancer 2012; 118:1701-1709.
10. National mastectomy and breast reconstruction audit. T.N.I. Centre, Editor; England. 2009. Disponible en: https://associationofbreastsurgery.org.uk/media/1083/nmbra-annual-report-2009.pdf

11. Mahmood U, Hanlon AL, Koshy M, et al. Increasing national mastectomy rates for the treatment of early stage breast cancer. Ann Surg Oncol 2013; 20:1436-1443.

12. Mansson D. Consenso Mexicano sobre diagnóstcio y tratamiento del cáncer de mamario. Septima edición. Elsevier 2017. Disponible en: http://www.consensocancermamario.com

13. Al-Ghazal SK, Fallowfield L, Blamey RW. Comparison of psychological aspects and patient satisfaction following breast conserving surgery, simple mastectomy and breast reconstruction. Eur J Cáncer 2000;36:1938-1943.

14. Eltahir Y, Werners LLCH, Dreise MM, et al. Which breast is the best? Successful autologous or alloplastic breast reconstruction: patient-reported quality-of-life outcomes. Plast Reconstr Surg J 2014; 135:43-50.

15. Elder EE, Brandberg Y, Bjorklund T, et al. Quality of life and patient satisfaction in breast cancer patients after immediate breast reconstruction: a prospective study. Breast 2005; 14: 201208.

16. Eltahir Y, et al. Quality-of-Life Outcomes between Mastectomy Alone and Breast Reconstruction: Comparison of Patient-Reported BREAST-Q and Other Health-Related Quality-of-Life Measures. Plast Reconstr Surg J 2013; 132:201-209.

17. King TA, Sakr R, Patil S, et al. Clinical management factors contribute to the decision for contralateral prophylactic mastectomy. J Clin Oncol 2011; 29:2158-2164.

18. Recalde M, Samudio M. Calidad de vida en pacientes con cáncer de mama en tratamiento oncológico ambulatorio en el Instituto de Previsión Social en el año 2010. Mem. Inst. Investig. Cienc. Salud. 2012; 10(2):13-29.

\title{
Comentario al artículo "Calidad de vida en reconstrucción mamaria post mastectomía. Aplicación del instrumento Breast-Q ${ }^{\circledR ”}$
}

\author{
Stefan DANILLA \\ Cirujano Plástico, Máster en Epidemiología Clínica, Hospital Clínico Universidad de Chile y Clínica Alemana, Santiago, Chile
}

Los autores presentan un estudio de cohortes en el cual se les aplicó el instrumento Breast- $Q^{\circledR}$ de calidad de vida $(\mathrm{CdV})$ asociado a la satisfacción con sus mamas en mujeres con secuelas de mastectomía por cáncer de mama en las cuales se realizó reconstrucción con tejido autólogo o protésico. Se trata de un estudio de cohorte transversal en el cual se preguntó en forma retrospectiva por la satisfacción con las mamas antes de la cirugía y en la misma llamada se preguntó por la satisfacción en el momento de la llamada.

De 52 paciente elegibles, 31 (59.6\%) contestaron a la encuesta descrita en la que se evidencia una mejoría significativa en la satisfacción con las mamas reconstruídas y en el bienestar sexual de las pacientes. Se presenta también la satisfacción postoperatoria en diferentes dominios del instrumento Breast- $Q^{\circledR}$ según el tipo de técnica de reconstrucción utilizada, sin realizar un análisis estadístico de las diferencias.
El instrumento Breast-Q ${ }^{\circledR}$ es un instrumento de medición de resultados, desde la perspectiva del paciente, ampliamente validado a nivel mundial y en varios idiomas, incluido el español. Tiene 3 módulos: 1) reconstrucción mamaria, 2) reducción mamaria y 3) aumento mamario; cada uno con un modulo preoperatorio y otro postoperatorio que pueden ser usados en forma longitudinal o transversal. Los resultados presentados por Gallegos Sierra y col. muestran una clara mejoría en la CdV de las pacientes sometidas a reconstrucción mamaria. Debido a algunos sesgos inherentes al diseño empleado y al tamaño de la muestra, no podemos determinar si esa mejoría se ve afectada por el tiempo de seguimiento, la edad de la paciente, el índice de masa corporal o el tipo de reconstrucción empleada ni el tipo de ablación oncológica. Idealmente, el instrumento Breast- ${ }^{\circledR}$ debiera ser aplicado de forma rutinaria a todas las pacientes sometidas a reconstrucción mamaria en el preoperatorio y luego, de forma estandari- 
zada, durante el seguimiento en uno o más momentos a lo largo del tiempo, por ejemplo a los 6 y 12 meses.

No obstante, los autores nos ilustran sobre que la aplicación de instrumentos de medición de resultados es factible y reproducible, obteniendo resultados comparables a estudios similares realizados en otras partes del mundo, que si bien nos refuerzan lo que como cirujanos plásticos ya sabemos, que con nuestras intervenciones mejoramos la calidad de vida de las personas, nos permiten además medir y cuantificar esa mejoría. El medir y cuantificar nos facilita además no solo comparar el antes y después, sino que con un número de pacientes suficiente, nos vale para determinar qué grupos de pacientes obtienen mayor mejoría, que técnicas son las

\section{Respuesta a comentario del Dr. S. Danilla}

Cuauhtly GALLEGOS SIERRA

En respuesta al comentario realizado a nuestro estudio, no queda más que agradecerle infinitamente al Dr. Danilla la atención prestada.

Si bien es cierto que en la literatura médica y en el ámbito de la Cirugía Plástica existe un sin número de publicaciones en relación a la reconstrucción mamaria y a las diferentes técnicas descritas al paso de los años, pocas veces se toman en cuenta las variables cualitativas entre las que se encuentra la calidad de vida de las pacientes que se han sometido a una reconstrucción postmastectomía. Estamos de acuerdo en que se necesitan estudios más extensos, con más eficaces y cuáles son los aspectos que los pacientes más valoran. Además, podremos con ellos presentar resultados medibles y objetivos a los administradores de salud, con los cuales podemos así no solo justificar nuestras intervenciones, sino exigir a las autoridades sanitarias que se destinen los recursos necesarios para completar la reconstrucción mamaria, la cual, a la luz de los resultados de este y otros estudios, mejora en forma sustancial y significativa la calidad de vida de las mujeres afectadas por cáncer de mama.

Felicito a los autores por su iniciativa y espero que los resultados de este estudio motiven a los cirujanos plásticos a aplicar de forma sistemática instrumentos de medición a sus pacientes.

una muestra mayor, para de esta forma considerar todas las variables señaladas tales como la edad y el índice de masa corporal. Definitivamente entendemos que lo estético es una variable de gran peso, pero por lo general se infravaloran otras variables como la sexualidad, el bienestar físico y el bienestar psicosocial. Consideramos por ello que nuestro estudio puede servir como evidencia para la toma de decisiones, principalmente en pacientes indecisas, que por los procesos oncológicos que han sufrido se encuentran temerosas de tomar una decisión que les lleve a un nuevo proceso quirúrgico. 